\title{
Voiding Dysfunction
}

\section{CHANGES IN BRAIN ACTIVITY FOLLOWING SACRAL NEUROMODULATION FOR URINARY RETENTION}

\author{
RANAN DASGUPTA,* HUGO D. CRITCHLEY, RAYMOND J. DOLAN AND CLARE J. FOWLER* \\ From the Department of Uro-Neurology, National Hospital for Neurology and Neurosurgery (RD, CJF), and Wellcome Department of \\ Imaging Neuroscience, Institute of Neurology, University College London (HDC, RJD), London, United Kingdom
}

\section{ABSTRACT}

Purpose: Sacral nerve stimulation (neuromodulation) can restore bladder sensation and the ability to void in women with urinary retention due to sphincter overactivity (Fowler's syndrome). Modulation of central afferent activity is considered critical to this therapeutic effect but the neural mechanisms are poorly understood. Therefore, we undertook a functional brain imaging study to determine how neuromodulation acts on brain centers involved in the representation and control of bladder function.

Materials and Methods: Eight patients with Fowler's syndrome and 8 healthy controls underwent brain imaging with positron emission tomography to identify regions of brain activity relating to the perception of bladder fullness and their modulation by sacral nerve stimulation.

Results: In healthy controls bladder fullness enhanced activity in brainstem (midbrain) and limbic cortical regions. Women with urinary retention showed no significant brainstem activity but did show enhanced limbic cortical activity when the bladder was full in the absence of neuromodulation. Neuromodulation restored a normal pattern of midbrain activity and decreased cortical activity in this group.

Conclusions: Our study provides novel neuroimaging evidence for the existence of abnormal interaction between brainstem and cortical centers in women with urinary retention. Furthermore, we have been able to show evidence that the therapeutic effect of sacral neuromodulation is achieved through restoration of activity associated with brainstem autoregulation and attenuation of cingulate activity.

KEY WoRDS: brain stem, diagnostic imaging, neurotransmitters, urinary retention

Sacral nerve stimulation (neuromodulation) is known to restore voiding function in patients with nonobstructive urinary retention, particularly in women with retention attributed to a functional overactivity of the urethral sphincter. ${ }^{1}$ Although there is increasing evidence that neuromodulation acts upon the afferent innervation in this group it is not clear at what level this occurs, and specifically which supraspinal centers may be involved.

Techniques of functional brain imaging have been used to identify the cerebral centers involved in performing specific visceral functions. One such technique is $\mathrm{H}_{2}{ }^{15} \mathrm{O}$ positron emission tomography (PET) scanning, which enables quantification of induced changes in relative activity within different brain regions. Enhanced activity of neurons within a particular region causes a local increase in regional cerebral blood flow which is measured from the distribution ${ }^{15} \mathrm{O}$ radiolabeled water given by bolus injection into the blood-

Submitted for publication February 13, 2005.

Nothing to disclose.

Study received local Ethics committee approval and Administration of Radioactive Substances Advisory Committee licensing.

Supported by the Wellcome Trust.

* Correspondence: Department of Uro-Neurology, National Hospital for Neurology, Queen Square, London WC1N 3BG (telephone: 0207-837-3611; FAX: 0207-813-4587; e-mail: ranandg@yahoo.co.uk).

Editor's Note: This article is the fourth of 5 published in this issue for which category 1 CME credits can be earned. Instructions for obtaining credits are given with the questions on pages 2430 and 2431 . stream. Previous PET studies have highlighted the involvement of brainstem and cortical regions, including midbrain (MB) and cingulate cortex (CC), in the control of bladder function during storage $\mathrm{e}^{2,3}$ and voiding. ${ }^{4,5}$

The present study uses PET scanning to explore brain processes observed in women with urinary retention due to sphincter overactivity and mechanisms through which neuromodulation may restore voiding function. The findings in patients are contrasted with those of a control group of healthy women. We hypothesized that therapeutic neuromodulation would act via afferent pathways at the level of the brainstem (including periaqueductal gray [PAG]), predicting abnormal PAG function would be restored to normal by neuromodulation.

\section{METHODS}

Subjects. The study was performed with local Ethics committee approval and Administration of Radioactive Substances Advisory Committee licensing. Eight healthy female volunteers (age range 40 to 62 years) and 8 women with retention (age range 39 to 52 years) successfully treated with sacral nerve stimulator implants were recruited $(2$ patients were excluded before analysis as they could not complete both scanning sessions). Subjects had an initial laboratory training session to familiarize them with the bladder sensation scoring system and to establish an approximate value for maximum bladder capacity. 
Neuroimaging data acquisition. Subjects were scanned on a Siemens ECAT PET scanner using a standard $\mathrm{H}_{2}{ }^{15} \mathrm{O}$ bolus technique (equivalent of $9 \mathrm{mCi}$ per scan). After an initial transmission scan to check subject positioning and determine the attenuation coefficient of the cerebral tissue, each subject underwent 12 PET scans with a minimum of 8 minutes between scans. Before each PET scan bladder volume was estimated noninvasively using a portable ultrasound scanner, and the subject rated the sensation of bladder fullness (scored 0 to 4, 0 representing no sensation of fullness, up to 4 representing desperate urge to void).

Each control subject underwent a 6 -scan session with the bladder empty (having voided just before the session), and 6 scans were obtained with the bladder full (filled by drinking water rapidly until a sensation of strong or desperate urge was reported). The order of having either a full or empty bladder was counterbalanced across subjects. Natural filling obviated the need for a catheter which might have introduced sensory artifact.

Each patient had 6 scans with the stimulator on and 6 with it deactivated (at least 3 days later to minimize carryover effects of neuromodulation). For each of the 6 scans, 3 were with a full and 3 with an empty bladder. Stimulator activation/deactivation and bladder empty/full scans were run in a counterbalanced order. With the stimulator switched off, patients were unable to void and typically described a sensation of suprapubic discomfort or heaviness (rather than urge to void) with increasing bladder volumes. With the stimulator activated, these subjects regained the feeling they would be able to micturate, but did not void while in the scanner.

Data analyses. Twelve scans per subject, corresponding to the distribution of regional cerebral blood flow during different states of bladder fullness (and in patients, neuromodulation) were preprocessed by spatial registration, spatial normalization, signal global normalization and smoothing using a $12 \mathrm{~mm}$ Gaussian kernel. Analyses were undertaken using Statistical Parametric Mapping (http://www.fil.ion.ucl.ac.uk/ $\mathrm{spm} / \mathrm{spm} 99 . \mathrm{html}$ ). Multiple linear regression group analysis was conducted on a voxel-by-voxel basis (voxel size $2 \mathrm{~mm}^{3}$ ) to identify activity related to experimental effects. We used an accepted threshold voxel-wide significance of $p<0.001$, uncorrected for multiple comparisons across whole brain. Nevertheless, we constrained our findings to clusters of 15 or more voxels in brainstem or limbic cortices (regions of interest, based on prior findings).

\section{RESULTS}

Subjective ratings. A significant correlation $(\mathrm{r}=0.88)$ was observed between measured bladder volume and desire to void (fig. 1 and Appendix 1) in healthy controls. Patients with retention in whom voiding was successfully restored by neuromodulation also demonstrated a similarly significant correlation $(r=0.86)$. During the study the empty bladder state generally corresponded to a rating (bladder filling sensation score) of 0 to 1 while the full state had a score of 3 to 4 .

Neuroimaging findings. We first identified the normal patterns of regional brain activity associated with bladder fullness in healthy female control subjects by statistically contrasting bladder full versus bladder empty scans. Having a full bladder was associated with enhanced activity within anterior midbrain in a region encompassing the substantia nigra (the source of ascending dopaminergic neurones to striatum and reciprocally connected with brainstem autonomic centers). A possible second brainstem activation was observed at the junction of pons/medulla although this was located at the periphery of the field of view of the scanner. The state of bladder fullness also activated cortical centers. Enhanced activity was observed in anterior "executive" and posterior "evaluative" regions of cingulate cortex during the combination of heightened urge to micturate and bladder fullness (fig. 2 and table 1).

In contrast to healthy controls, patients with retention did not show enhanced activation of midbrain regions during bladder fullness in the absence of sacral nerve stimulation. However, they did show activation of anterior and posterior cingulate cortex. The location of this activity overlapped with that seen in controls (fig. 3 and table 2). With the stimulator switched on, patients with a full bladder showed activity in the brainstem, mainly in the midbrain, similar to healthy controls (fig. 4 and table 3). Appendix 2 summarizes the findings for these conditions.

\section{DISCUSSION}

Neuromodulation by sacral nerve stimulation is an effective intervention for the treatment of voiding dysfunction, and paradoxically used for urinary retention and urgencyfrequency/urge incontinence. The mechanism of neuromodulation is uncertain but likely to involve afferent pathways to the brain regulating central control of micturition. We report the findings of a functional brain imaging study exploring, for the first time, central effects of neuromodulation in urinary retention, and provide novel evidence for a brain correlate (attenuated brainstem activity) of altered interoceptive and visceromotor function in women with sphincter overactivity. Moreover, our findings suggest that neuromodulation achieves its therapeutic effect at the level of functional interactions between midbrain and limbic cingulate cortex.

We demonstrate that bladder fullness in healthy females is associated with enhanced activity in brainstem (midbrain and pons), and anterior and posterior cingulate cortices. In the absence of neuromodulation, women with retention due to sphincter overactivity lack the normal pattern of midbrain activation, but show fullness related activity enhancement of anterior and posterior cingulate cortices. However, when sacral neuromodulation is switched on, the fullness related activity in the brainstem (midbrain) returns to normal and even surpasses that seen in healthy women, whereas cingulate activity is markedly attenuated. Theoretical arguments suggest that brainstem regions are involved in primary autoregulatory representations (for example of bladder volume) whereas cingulate cortices contribute to second order contextual representations of these internal visceral processes ${ }^{6,7}$ and the interactions between them are essential for behavioral control. Our observations identify key cerebral centers involved in normal and abnormal control of micturition and highlight the role of afferent central pathways, modulated by sacral nerve stimulation, in restoring bladder and sphincter function in urinary retention.

Brainstem. Brainstem regions provide the first level of supraspinal control of urinary function. In the dorsal midbrain region, the PAG is considered to act as a relay center,

\section{Urge score versus bladder volume - healthy controls}

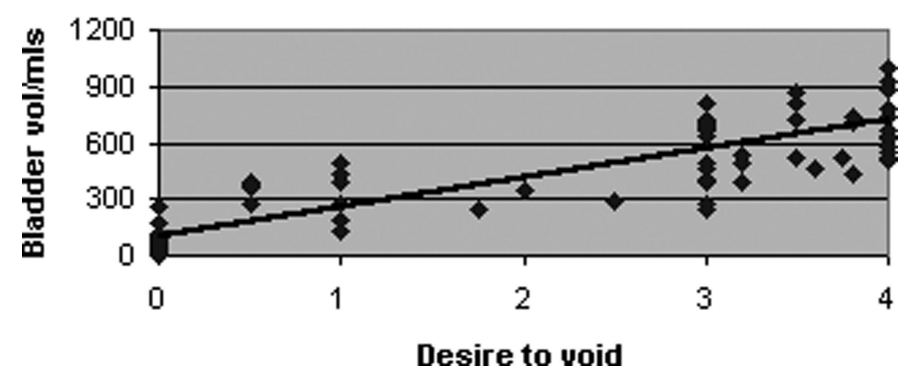

FIG. 1. Sensation of desire to void (range 0 to 4 ) versus bladder volume. 

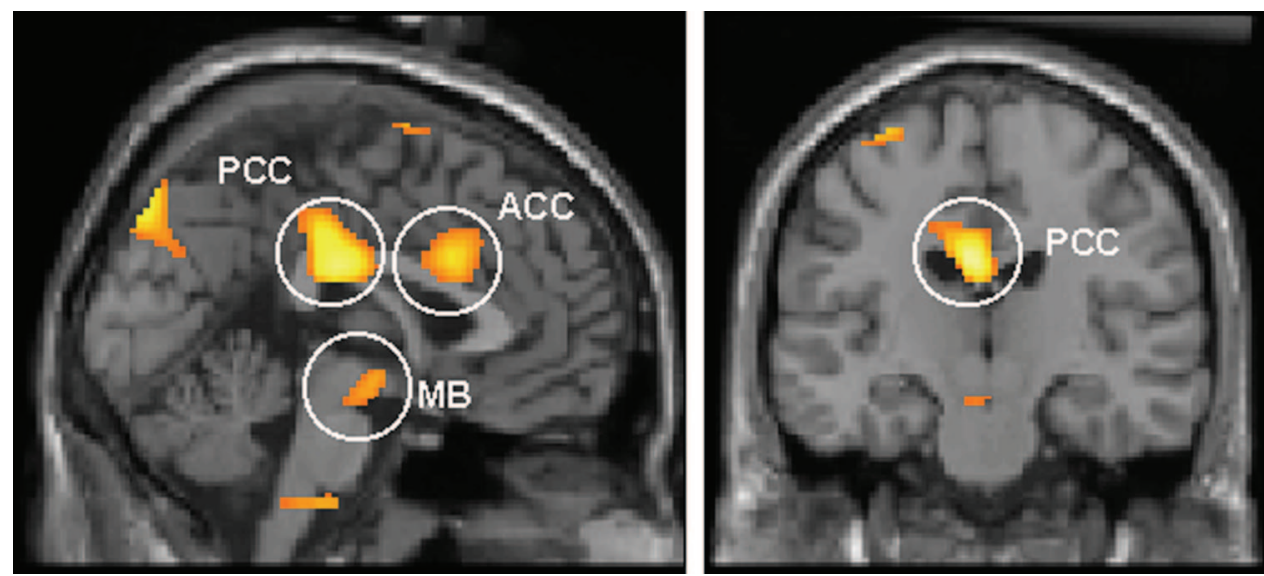

Fig. 2. Localized foci of brain activation in healthy female volunteers with full bladder (compared to empty bladder). MB, midbrain.

TABLE 1. Localized foci of brain activation

\begin{tabular}{|c|c|c|c|c|}
\hline \multirow[t]{2}{*}{ Brain Region } & \multicolumn{3}{|c|}{$\begin{array}{c}\text { Peak Activation } \\
\text { Position* }\end{array}$} & \multirow[t]{2}{*}{ Z Score } \\
\hline & $\mathrm{X}$ & $\mathrm{Y}$ & $\mathrm{Z}$ & \\
\hline MB & 2 & -10 & -12 & 3.94 \\
\hline $\mathrm{ACC}$ & -4 & 18 & 28 & 3.38 \\
\hline PCC & -4 & -22 & 30 & 6.16 \\
\hline
\end{tabular}
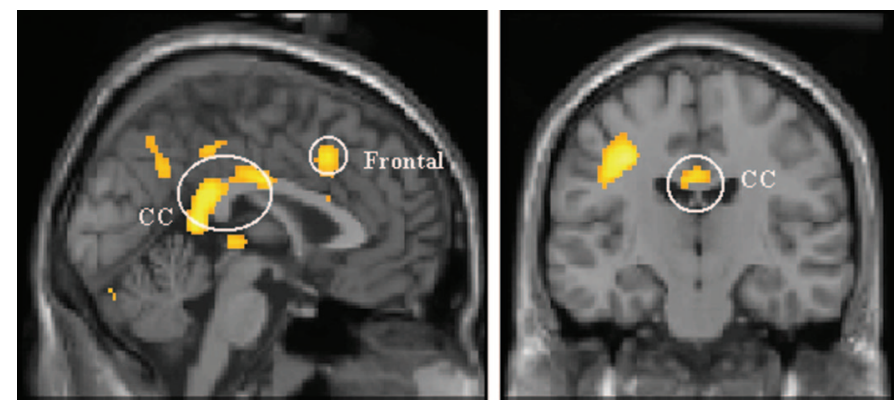

FIG. 3. Brain activation in women with urinary retention at baseline (with stimulator off).

TABLE 2. Brain activation in women with urinary retention at baseline (with stimulator off)

\begin{tabular}{lrrrr}
\hline \multirow{2}{*}{ Brain Region } & \multicolumn{3}{c}{ Peak Activation Position } \\
& \multicolumn{3}{c}{$(\mathrm{mm})$} & Z Score \\
\cline { 2 - 4 } & $\mathrm{X}$ & $\mathrm{Y}$ & $\mathrm{Z}$ & \\
\hline Cingulate & 4 & -12 & 30 & 3.87 \\
Frontal cortex (lt) & 18 & 22 & 34 & 3.94 \\
\hline
\end{tabular}

Uncorrected height threshold, $\mathrm{p}<0.001$.

receiving afferent input from the sacral spinal cord ${ }^{8}$ and thereby communicating with the efferent pontine micturition center. ${ }^{9}$ In turn, the pontine micturition center projects to sacral parasympathetic preganglionic neurones. While our findings of enhanced midbrain activity during states of bladder fullness in healthy controls is broadly consistent with animal and neuroimaging studies highlighting the role of $\mathrm{PAG}^{2,3,10,11}$ it is noteworthy that our results implicate a slightly more anterior midbrain region. The representation of bladder fullness by midbrain activity may therefore not be solely localized to the PAG, but instead may be a more diffuse activation encompassing other midbrain sites such as the substantia nigra.
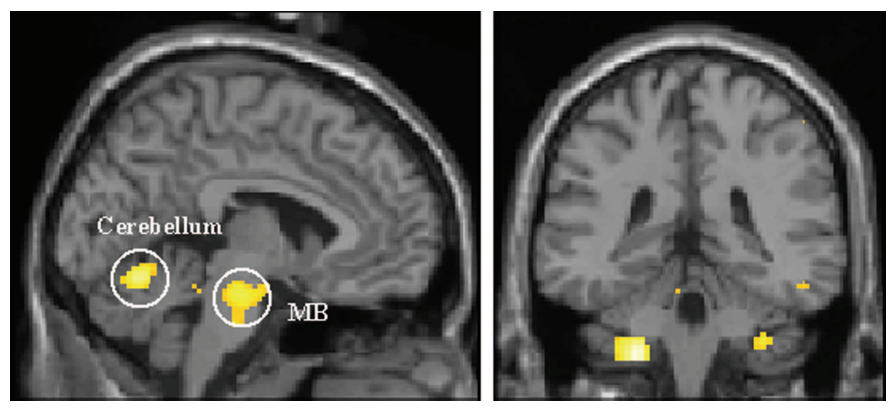

Fig. 4. Brain activity in women with Fowler's Syndrome and stimulator activated.

TABLE 3. Brain activity in women with Fowler's syndrome and stimulator activated

\begin{tabular}{lcccc}
\hline \multirow{2}{*}{ Brain Region } & \multicolumn{3}{c}{ Peak Activation Position } & \\
\cline { 2 - 3 } & $\mathrm{X}$ & $\mathrm{Y}$ & $\mathrm{Z}$ & Z Score \\
\hline MB & 2 & -12 & -24 & \\
Cerebellum & -2 & -68 & -14 & 4.03 \\
\hline Uncorrected height threshold, $\mathrm{p}<0.001$. & & \\
\hline
\end{tabular}

The substantia nigra and ventral tegmental area are the origin of dopaminergic projections from midbrain to striatum and cortex. Functionally these pathways facilitate motor function and drive motivational behaviors, both of which are affected in Parkinson's disease, for example. Effective micturition demands a motivational representation (urge) and volitional change in sphincter muscular tonicity. Neurones located in substantia nigra and ventral tegmentum respond to bladder filling and help determine the biphasic micturition reflex. ${ }^{12}$ The precise localization of putative nuclei accounting for observed midbrain activation remains uncertain within the spatial resolution of PET and more circumscribed issues with brainstem imaging. Nevertheless, our data suggest that midbrain involvement in micturition control extends beyond the PAG.

A key observation in the retention group was the restoration of midbrain and pontine activity by sacral neuromodulation, which is consistent with an afferent effect on the midbrain relay that links brainstem (PAG) and cortical (cingulate) bladder representations to systems supporting programmed motor behavior and motivational control. More simply, neuromodulation could restore voiding function by resetting brainstem function (midbrain, in particular), which also remains under influence from higher cortical centers. 
Cingulate cortex. Neuromodulation, which clinically restored desire and ability to void, induced a striking attenuation of the normal pattern of anterior and posterior cingulate activation in patients, suggesting that whatever is represented by this limbic activity may be inhibiting the ability to void through descending control. This hypothesis fits with a widespread notion within human psychology that executive control (including suppressing urges or emotions), volitional reasoning and other high cognitive processes are mediated in part by the anterior cingulate. Lesions of anterior cingulate may decrease motivation. The term "visceral brain" highlights the functional and anatomical relationship between limbic regions and brainstem centers involved in visceral control. Anterior cingulate cortex (ACC) activity is enhanced by painful, ${ }^{13,14}$ emotional, ${ }^{15}$ cognitive ${ }^{16}$ and visceral stimulation. ${ }^{17}$ The ACC is likely to be a part of a network of brain centers involved in the modulation of autonomic response to noxious stimuli. It is reciprocally connected to other cortical and brainstem centers including the amygdala, hypothalamus, PAG, substantia nigra and ventral tegmental area. Extensive damage to frontal regions involving ACC may result in profound motivational impairment or in urge incontinence. ${ }^{18}$ More recent functional brain imaging studies have shown changes in activity of the ACC with urine storage ${ }^{2,3}$ and micturition. ${ }^{4,19}$ These observations are consistent with a proposal that the ACC supports an integration of visceral afferent information with internal motivational states and external behavioral cues to drive facilitatory autonomic and motor responses for adaptive behavior. ${ }^{20}$ In this patient group neuromodulation may interfere with the hierarchical representation of afferent visceral information, altering the interaction between cingulate and midbrain centers.

The activity of posterior cingulate cortex (PCC) paralleled that of ACC in this study, and it is noteworthy that PCC stimulation in animal experiments can interrupt micturition. ${ }^{21}$ However, PCC and ACC may have complementary roles in the context of bladder filling. It is suggested that whereas the ACC has a predominantly executive function, the PCC has been characterized as more evaluative. ${ }^{22}$ There is some empirical support for this representational role from observations of autonomic states, ${ }^{6}$ neuropathic pain ${ }^{23}$ and emotional processing. ${ }^{24}$ However, the functional role of PCC remains elusive and a tentative interpretation of our data is that the PCC may support a motivational representation of bladder fullness, ie to empty the bladder before becoming too full.

\section{CONCLUSIONS}

Our findings show that urinary control depends on interactions of several brain centers including the cingulate cortex, midbrain and pons, with evidence that sacral neuromodulation has central effects on these areas. Importantly, our study indicates that anterior midbrain activation is necessary for perceiving bladder fullness, and details the functional interaction of ACC and PCC with brainstem centers in this role.

Karen Berkley provided discussions and scan, the radiographers assisted with the scanning, and Collette Haslam and Gwen Gonzales assisted with the subjects.

APPENDIX 1: SENSATION OF DESIRE TO VOID (0-4) VERSUS BLADDER VOLUME

No sensation of fullness

Initial sensation of filling

Sensation of bladder fullness

Strong desire to void

Desperate desire to void

Regression value $\mathrm{r}=0.88$
APPENDIX 2: SUMMARY OF DIFFERENCES IN ACTIVITY IN BRAIN ACTIVITY IN DIFFERENT CONDITIONS

\begin{tabular}{lcc}
\hline & $\begin{array}{c}\text { Brainstem } \\
\text { activity }\end{array}$ & $\begin{array}{c}\text { Cingulate } \\
\text { cortex activity }\end{array}$ \\
\hline Controls & Yes & Yes \\
Patient stimulator off & No & Yes \\
Patient stimulator on & Yes & No \\
\hline
\end{tabular}

\section{REFERENCES}

1. DasGupta, R. and Fowler, C. J.: The management of female voiding dysfunction: Fowler's Syndrome-a contemporary update. Curr Opin Urol, 13: 293, 2003

2. Athwal, B. S., Berkley, K. J., Hussain, I., Brennan, A., Craggs, M., Sakakibara, R. et al: Brain responses to changes in bladder volume and urge to void in healthy men. Brain, 124: 369, 2001

3. Matsuura, S., Kakizaki, H., Mitsui, T., Shiga, T., Tamaki, N. and Koyanagi, T.: Human brain region response to distention or cold stimulation of the bladder: a positron emission tomography study. J Urol, 168: 2035, 2002

4. Blok, B. F., Willemsen, A. T. and Holstege, G.: A PET study on brain control of micturition in humans. Brain, 120: 111, 1997

5. Blok, B. F., Sturms, L. M. and Holstege, G.: Brain activation during micturition in women. Brain, 121: 2033, 1998

6. Critchley, H. D., Mathias, C. J. and Dolan, R. J.: Neuroanatomical basis for first- and second-order representations of bodily states. Nat Neurosci, 4: 207, 2001

7. Damasio, A.: Feelings of emotion and the self. Ann N Y Acad Sci, 1001: 253, 2003

8. Keay, K. A., Clement, C. I., Owler, B., Depaulis, A. and Bandler, R.: Convergence of deep somatic and visceral nociceptive information onto a discrete ventrolateral midbrain periaqueductal gray region. Neuroscience, 61: 727, 1994

9. Blok, B. F., De Weerd, H. and Holstege, G.: Ultrastructural evidence for a paucity of projections from the lumbosacral cord to the pontine micturition center or M-region in the cat: a new concept for the organization of the micturition reflex with the periaqueductal gray as central relay. J Comp Neurol, 359: 300, 1995

10. Kruse, M. N., Noto, H., Roppolo, J. R. and de Groat, W. C.: Pontine control of the urinary bladder and external urethral sphincter in the rat. Brain Res, 532: 182, 1990

11. Taniguchi, N., Miyata, M., Yachiku, S., Kaneko, S., Yamaguchi, S. and Numata, A.: A study of micturition inducing sites in the periaqueductal gray of the mesencephalon. J Urol, 168: 1626, 2002

12. Winge, K. and Fowler, C.: Bladder dysfunction in Parkinsonian disease and parkinsonian syndromes. Unpublished data

13. Coghill, R. C., Talbot, J. D., Evans, A. C., Meyer, E., Gjedde, A., Bushnell, M. C. et al: Distributed processing of pain and vibration by the human brain. J Neurosci, 14: 4095, 1994

14. Rainville, P.: Brain mechanisms of pain affect and pain modulation. Curr Opin Neurobiol, 12: 195, 2002

15. George, M. S., Ketter, T. A., Parekh, P. I., Horwitz, B., Herscovitch, P. and Post, R. M.: Brain activity during transient sadness and happiness in healthy women. Am J Psychiatry, 152: 341, 1995

16. Bush, G., Luu, P. and Posner, M. I.: Cognitive and emotional influences in anterior cingulate cortex. Trends Cogn Sci, 4: 215,2000

17. Hobday, D. I., Aziz, Q., Thacker, N., Hollander, I., Jackson, A. and Thompson, D. G.: A study of the cortical processing of ano-rectal sensation using functional MRI. Brain, 124: 361, 2001

18. Andrew, J. and Nathan, P. W.: Lesions on the anterior frontal lobes and disturbances of micturition and defaecation. Brain, 87: 233,1964

19. Nour, S., Svarer, C., Kristensen, J. K., Paulson, O. B. and Law, I.: Cerebral activation during micturition in normal men. Brain, 123: 781, 2000

20. Critchley, H. D., Mathias, C. J., Josephs, O., O’Doherty, J., Zanini, S., Dewar, B. K. et al: Human cingulate cortex and autonomic control: converging neuroimaging and clinical evi- 
dence. Brain, 126: 2139, 2003

21. Gjone, R.: Excitatory and inhibitory bladder responses to stimulation of 'limbic', diencephalic and mesencephalic structures in the cat. Acta Physiol Scand, 66: 91, 1966

22. Vogt, B. A., Finch, D. M. and Olson, C. R.: Functional heterogeneity in cingulate cortex: the anterior executive and posterior evaluative regions. Cereb Cortex, 2: 435, 1992
23. Hsieh, J., Belfrage, M., Stone-Elander, S., Hansson, P. and Ingvar, M.: Central representation of chronic ongoing neuropathic pain studied by positron emission tomography. Pain, 63: 225,1995

24. Maddock, R. J., Garrett, A. S. and Buonocore, M. H.: Posterior cingulate cortex activation by emotional words: fMRI evidence from a valence decision task. Hum Brain Mapp, 18: 30, 2003 